\title{
In vivo blood flow and wall shear stress measurements in the vitelline network
}

\author{
C. Poelma $\cdot$ P. Vennemann $\cdot$ R. Lindken $\cdot$ \\ J. Westerweel
}

Received: 14 November 2007 / Revised: 25 January 2008/ Accepted: 3 February 2008/Published online: 28 February 2008

(C) The Author(s) 2008

\begin{abstract}
The wall shear stress plays a key role in the interaction between blood flow and the surrounding tissue. To obtain quantitative information about this parameter, velocity measurements are required with sufficient spatial (and temporal) resolution. We present a methodology for the determination of the wall shear stress in vivo in the vitelline network of a chick embryo. Velocity data is obtained by microscopic particle image velocimetry using correlation ensemble averaging; the latter is used to increase the signal-to-noise ratio of the measurements. The temporal evolution of the pulsatile flow is reconstructed by sorting the image pairs based on a phase estimate. From these flow measurements, the wall shear stress can be derived either directly from the magnitude of the gradients or from fits to velocity profiles. Both methods give results that are in good agreement with each other, while the former method is significantly easier to implement. For more accurate studies, the full threedimensional velocity field may be required. It is demonstrated how this velocity field can be obtained by scanning the measurement volume.
\end{abstract}

\section{Introduction}

During the development of the circulatory system, blood flow and vascular changes are strongly coupled. Hemodynamic forces acting on the blood vessels result in a

C. Poelma $(\varangle) \cdot$ P. Vennemann · R. Lindken · J. Westerweel Laboratory for Aero and Hydrodynamics,

Delft University of Technology, Leeghwaterstraat 21,

2628 CA Delft, The Netherlands

e-mail: C.Poelma@tudelft.nl constantly changing geometry, due to the plasticity of the vessel walls. Of these hemodynamic forces, the wall shear stress (WSS) has seen a strong increase in attention over the last decade. The endothelial cells that line the inner wall of blood vessels react in various ways to changes in the shear stress, e.g. the formation of cilia in low shear zones (Van der Heiden et al. 2006) and the expression of certain growth factors (Topper and Gimbrone 1999; McCormick et al. 2001). These responses are crucial in the development of the cardiovascular system (Hierck et al. 2008), as well as for the proper functioning of an organism (Reneman et al. 2006; Resnick et al. 2003). Note that the underlying mechanisms at cellular level, the so-called mechano- and chemotransduction, is a topic of current research, see e.g. Weinbaum et al. (2003) and Janmey and McCulloch (2007). WSS also plays a role in the etiology of a number of diseases: for instance, regions with low or oscillating WSS have been linked to atherosclerosis-the deposition of vulnerable plaque at the vessel wall (Ku et al. 1985).

While the significance of WSS is well accepted, it is a notoriously difficult quantity to actually measure (Vennemann et al. 2007). Conventionally it is estimated, for instance by assuming that the flow can be described as Poiseuille flow. Assuming a parabolic flow in a given vessel, the WSS $(\tau)$ can then be found using the flow rate $(q)$, vessel diameter $(d)$ and dynamic viscosity $(\eta)$ :

$\tau=\frac{4 \eta q}{8 \pi d^{3}}$

The value for $q$ can either be estimated from ultrasound Doppler measurements or from videomicroscopy (by e.g. measuring the displacement of red blood cells). Obviously, the validity of the assumptions of Poiseuille flow can be debated in cardiovascular flows. Effects of pulsatile flow, 
expressed in the Womersley number $\alpha=d \sqrt{2 \pi f \rho / \eta}$ (with $f$ the frequency of pulsation and $\rho$ the fluid density), are ignored. A high value of $\alpha$ results in a flattening of the velocity profile (Batchelor 1974; McDonald 1974). Similarly, it is assumed that the flow after e.g. a bifurcation instantaneously returns to parabolic flow. The entrance effects, expressed in the required length needed for development of a parabolic flow profile ( $L / D$, with $D$ the vessel diameter), are dependent on the Reynolds number (White 2007). In combination with a strongly curved geometry this can lead to asymmetric flow profiles that deviate significantly from the classic parabola (Vennemann et al. 2006a). Finally, all hemorheological aspects are ignored in the equation above: it is well-known that the formed elements of the blood (especially the red blood cells) are not distributed homogeneously over the vessel diameter (Popel and Johnson 2005). Due to for instance the so-called Fahræus-Lindqvist effect the viscosity can vary over the profile. This effect becomes more relevant when the vessel diameter approaches the size of individual red blood cells (with a typical size of 6-8 $\mu \mathrm{m}$ ). Additionally, blood exhibits shear-thinning behavior (Chien 1970).

An alternative to Eq. 1 is to use the definition of the WSS, i.e. the derivative of the fluid velocity $u$ perpendicular to the vessel wall (represented as wall-normal unit vector $n$ ):

$\tau=\eta \frac{\partial u}{\partial n}$

This seemingly straightforward approach does not need the assumptions of Poiseuille flow. However, it requires very detailed flow velocity measurements close to the wall in order to estimate the spatial derivative. In the current study, we investigate the possibilities of such accurate measurements in vivo in the so-called vitelline network of a chick embryo (see Fig. 1) using micro-PIV. The extraembryonic vitelline network ${ }^{1}$ ensures that nutrients from the yolk sac are transported to the embryo. Chick embryos are a commonly used model system for the early development of the human cardiovascular system, as it closely mimics the development of the human heart and circulation. The chick embryo, and in particular the vitelline network, is an accessible system to study the formation and adaptation of vessels (vasculogenesis, angiogenesis, etc.). Examples of these studies include the role of flow on the differentiation between arteries and veins (Le Noble et al. 2004) and the influence of cardiac inflow on the morphogenesis of the heart (Hogers et al.

\footnotetext{
1 The vitelline network consists of the left and right omphalomesenteric arteries and the anterior and posterior omphalo-mesenteric veins. For a detailed description and biological background, we refer to Bellairs and Osmond (2005).
}

1997). Both these studies relied on a qualitative description of the flow, i.e. flow visualization. While this gives valuable insight in the mechanisms at hand, it limits a more exact modeling. A previous study of the cardiac cycle of an embryonic heart (Vennemann et al. 2006a) showed that in vivo blood flow measurements using micro-PIV can greatly contribute to a more quantitative understanding.

Recently, a similar study of the flow in the chick vitelline network has been published by Lee et al. (2007). In this study, the authors used red blood cells as flow tracers. This approach assumes that these red blood cells follow the flow of plasma accurately-an assumption that will most likely not be valid in the case of the small blood vessels under consideration (Vennemann et al. 2007). For an approximation of the the mean flow rate, tracking red blood cells at the centerline is acceptable. For detailed velocity estimates near the wall, as required for Eq. 2, this method may not give accurate results: Sugii et al. (2005) have shown in vitro that there is a velocity difference between red blood cells and plasma. The current study provides a refinement by using small fluorescent tracer particles. Furthermore, we will focus on shear stress measurements and show the possibilities of three-dimensional flow measurements.

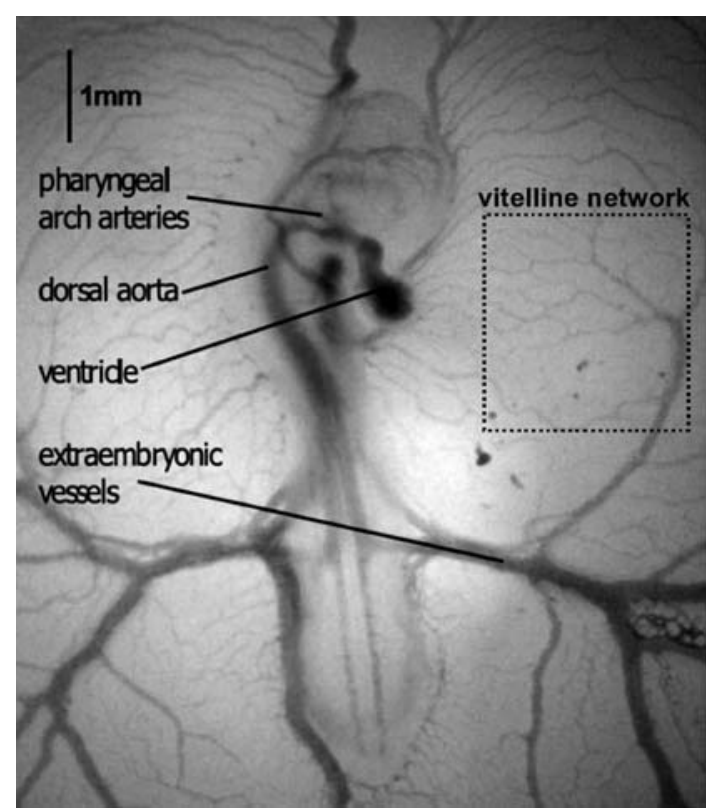

Fig. 1 A chick embryo (Hamburger and Hamilton stage 15, approximately $50 \mathrm{~h}$ after incubation) showing the extra-embryonic blood vessels that form an intricate network through the yolk sac around the embryo. This network, known as the vitelline system, transports nutrients from the yolk to the growing embryo (adapted from Vennemann et al. 2006a) 


\section{Methodology}

\subsection{Materials}

Fertilized chicken eggs (Gallus gallus) are incubated until they reach developmental stage HH 18 (Hamburger and Hamilton 1951), corresponding to approximately three days. A small window is carefully removed from the egg shell to allow optical access to the embryo. During measurements, the egg is kept at a constant temperature of $37^{\circ} \mathrm{C}$. The egg is placed under a combined stereo/mono fluorescence microscope (Leica MZ 16 FA), as shown in Fig. 2. The stereo mode is used for preparation of the experiments only, e.g. for tracer injection. The microscope is fitted with a PCO Sensicam QE camera $(1,376 \times 1,040$ pixels, using $2 \times 2$ binning) and an apochromatic objective with $5 \times$ magnification (Leica FluoCombi III). Illumination is done with a diode-pumped Nd:YLF laser (New Wave Pegasus). Note that the motivation of using this laser was the relatively long pulse duration ( $40 \mathrm{~ns}$ ), which increases the yield of the fluorescent dye in the tracer particles by a factor of 2 to 3 as compared to a conventional Nd:YAG laser. The facility is controlled using a PC running DaVis 7 (LaVision $\mathrm{GmbH}$ ), which is also used for data acquisition and storage. As tracer particles, $1 \mu \mathrm{m}$ polystyrene spheres are used containing a fluorescent dye (Rhodamine 6G, Microparticles $\mathrm{GmbH}$ ). Due to a poly-ethylene glycol (PEG) coating, the particles are bio-inert or 'stealth': they are circulating throughout the embryo without any detectable adverse effects for the duration of several hours. Penetration of the endothelial layer is minimal, so that they do not enter the

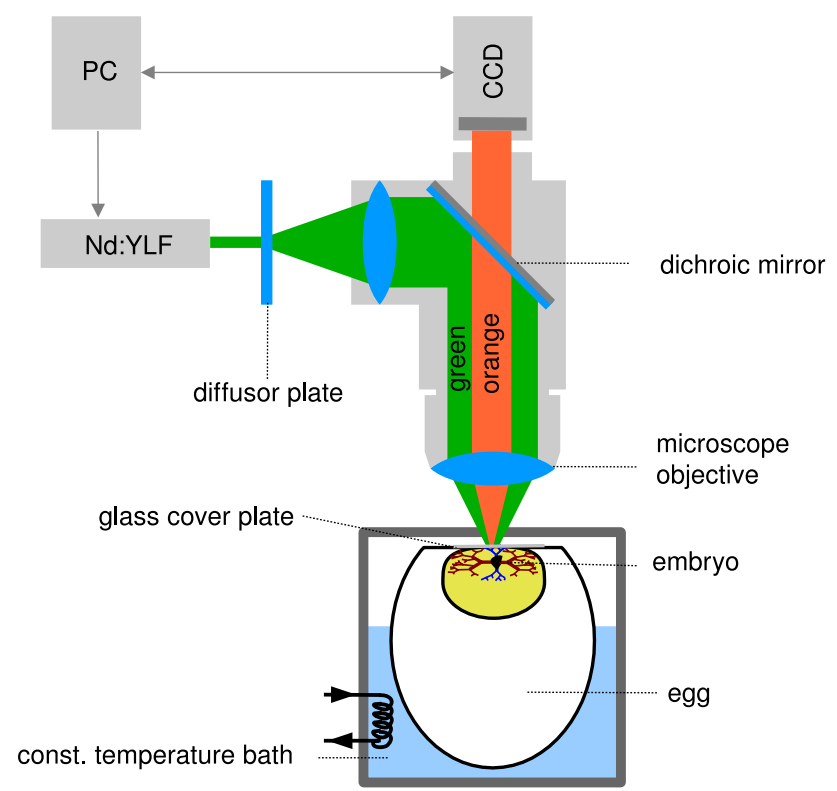

Fig. 2 Schematic representation (not to scale) of the measurement set-up. See text for details yolk. The particles are neutrally buoyant and have a (Stokes) response time smaller than $1 \mu \mathrm{s}$, indicating that they are expected to follow even the fastest fluid fluctuations. The particles are injected, suspended in a phosphate-buffered saline (PBS) solution, in one of the vitelline veins by means of a micro injector system and a glass micro needle with a tip diameter of a $5-10 \mu \mathrm{m}$. The total injection volume, calculated a posteriori from the observed tracer particle image density, never exceeded $0.1 \mu \mathrm{l}$. This is small enough to avoid significant effects for the cardiovascular system (Wagman et al. 1990). The effect of the tracer particles on the viscosity - the effective viscosity of the suspension, see e.g. Landau and Lifshitz (1987)-is negligible due to the low volume fraction: $0.02 \%$ based on a total circulation volume at this stage of $40 \mu \mathrm{l}$ (Kind 1975). Within a few minutes, the tracer particles are distributed over all vessels both inside and outside the embryo.

\subsection{Recording strategy and work-flow}

Ideally, the measurements should be able to document the transient phenomena of the pulsatile flow, which has a typical frequency of $2 \mathrm{~Hz}$. The data rate of the measurement system is (depending on the recording method), typically $10 \mathrm{~Hz}$. This would mean that only 5 frames per cycle can be obtained. An additional complication is the fact that the image quality is not optimal (see Fig. 3 for an example raw PIV image). Due to the blurring effect of outof-focus tissue and a lack of tracer particles, initial PIV results had insufficient quality. Typical values for the tracer particle density (estimated by visual inspection of a series of raw images) were 0.003 particle/pixel. Adding more particles would be possible, but then the injection volume might become large enough to start influencing the cardiac performance.

To overcome this complication, it was decided to use the following strategy: (1) data is recorded continuously, (2) the cardiac phase is determined for each image pair, (3) the image pairs are sorted based on their phase, (4) an ensemble correlation average is calculated for each phase group. This approach results in an increase both in data quality and in (reconstructed) temporal resolution. With an ensemble of $n$ image pairs, the effective tracer particle density in the ensemble correlation increases by a factor $n$. This ensemble averaging approach assumes that the flow is quasi-stationary and that cycle-to-cycle differences are small. This is checked post hoc in two steps: movements of the embryo are detected and, if possible, corrected in an image registration step to ensure a similar field-of-view. Changes in the temporal velocity pattern are evaluated in the image sorting step. More detail about the work-flow, including these two steps, is given below and the process is shown schematically in Fig. 4. 
Fig. 3 Example of a raw image (left) and a close-up of the image after subtraction of the mean image (right). The gray values have been inverted for clarity

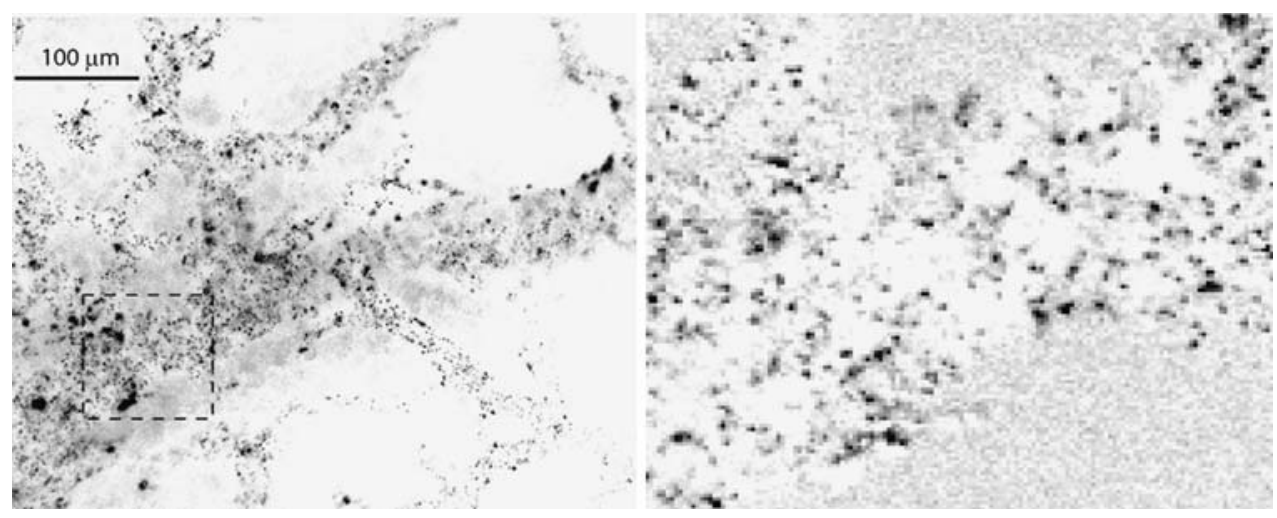

\subsubsection{Image recording}

Typically 1,000 image pairs are recorded for each measurement. These measurements are not phase-locked with the heart beat. Phase-locking is possible, as shown by Vennemann et al. (2006b), using e.g. a Doppler ultrasound velocimeter to trigger the PIV acquisition at a given moment in the cycle. However, this would have increased the measurement time by an order of magnitude, as only one image pair per cardiac cycle (which typically lasts $0.5 \mathrm{~s}$ ) can be recorded. In this case, changes in the embryo could no longer be ignored during recording data sets of sufficient length. Additionally, setting up the ultrasound probe is very time-consuming.

The data acquisition can be done either using singleframe images at $20 \mathrm{~Hz}$ ('cinematographic') or at $10 \mathrm{~Hz}$

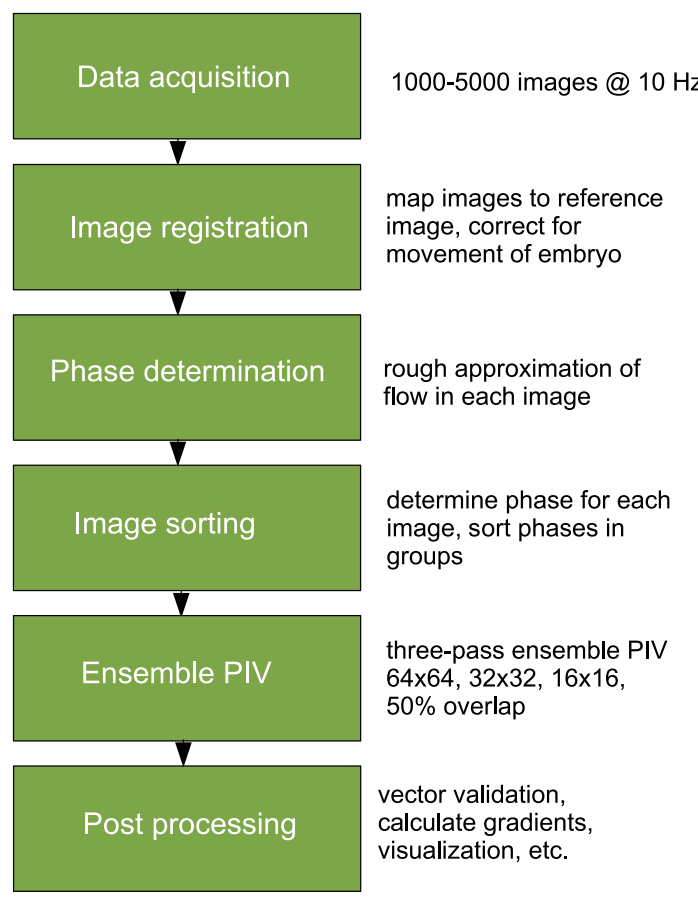

Fig. 4 Schematic representation of the workflow, see text for details using double-frame images ('cross-correlation' mode). The latter has the obvious advantage that the delay time between the laser pulses $(\Delta \mathrm{T})$ can be varied, while it is fixed at $\Delta T=1 / 20 \mathrm{~s}$ in the former. The choice for the recording mode depends on the magnification and the specific vessel network under consideration. In practice the $10 \mathrm{~Hz}$ recording rate of the double-frame mode was found to be sufficient to determine the cardiac phase (discussed below), so this more flexible recording mode is used. The value of $\Delta T$ is optimized so that the maximum displacement of the tracer particles at the peak of the velocity cycle (systole) was 10-12 pixels. Increasing the value of $\Delta T$ not only leads to larger tracer displacements, but obviously also to larger absolute differences in the displacement within each interrogation area (see Westerweel 2008 for a discussion). The latter are found to be the limiting factor for good PIV results in the current experiment. ${ }^{2}$ Typical values for $\Delta T$ of $3,000-5,000 \mu$ s were found to yield good results. Note that for each experiment a single $\Delta T$ is used that is a compromise to get good results throughout the entire cardiac cycle. Because at the moment of recording it is not known which velocities are to be expected, implementing a varying, optimized $\Delta T$ for each image pair is not feasible (this is in contrast to the aforementioned phase-locked measurements using a Doppler ultrasound velocimeter).

\subsubsection{Image registration}

The data acquisition of one measurement takes approximately 2 min. During this time, the embryo occasionally moves slightly. Therefore, image registration is needed to compensate for these movements. The first image is used as reference image and all subsequent images are mapped onto this image. To determine the required image transformation, the disparity between the images is determined

2 The quality of the PIV result was judged by the number of outliers in the final result inside the vessels only (i.e. disregarding vectors inside tissue); the percentage of outliers was less than 5\%. 
by local cross-correlation using $96 \times 96$ pixels interrogation windows with $50 \%$ overlap, covering the entire raw image (Willert 1997). Note that the tracer particles do not contribute to this particular cross-correlation analysis, as their location is completely uncorrelated due to the relatively large time separation between the image pairs. A second-order polynomial fit is performed using this disparity data. Subsequently, the image is corrected using an affine transformation based on this polynomial fit. In general, the corrections needed were small (i.e. a uniform translation of one or two pixels). Nevertheless, this step is critical for the determination of the average gray value image that will be subtracted from each image before PIV processing. This subtraction effectively removes bright spots mainly caused by clusters of tracer particles that occasionally adhere to the vessel walls during the measurement (see Fig. 3).

\subsubsection{Phase determination}

The phase of each image pair is determined by evaluating the displacement in a relatively large interrogation area $(96 \times 96$ pixels or larger if needed), similar to the approach used by Vennemann et al. (2006b). The location of the interrogation area is chosen within a large vessel, so that a strong signal (or dynamic range, to be more precise) is expected. While such a large interrogation area will yield information that is a spatial average over a relatively large region, it provides us with a robust estimate of the displacement. During the cardiac cycle, only one 'peak' in the blood velocity is expected. As can be seen in Fig. 5, this peak (systole) can clearly be observed. The figure shows the displacement (in arbitrary units) in a large region in a

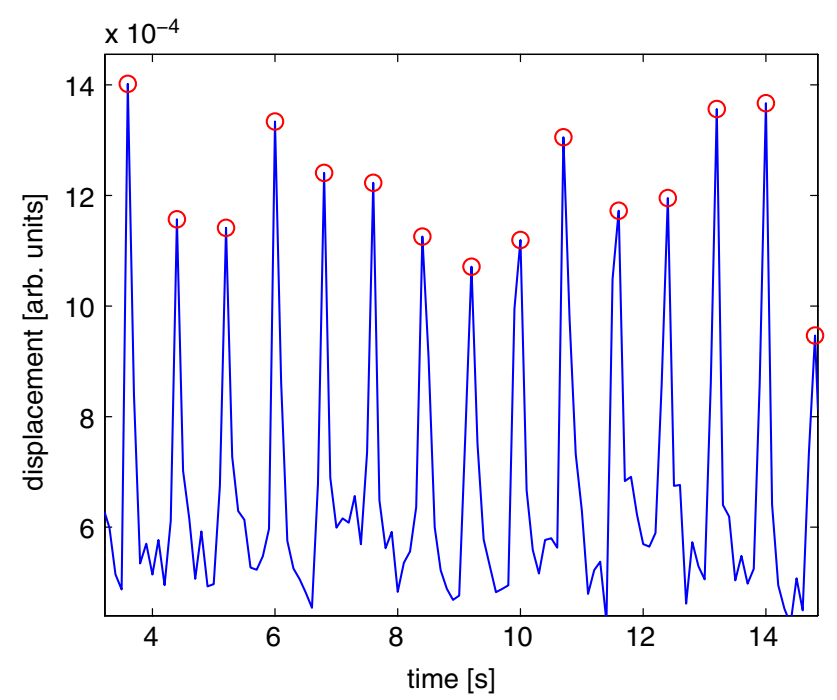

Fig. 5 'Quick-and-dirty' displacement estimation for the image series and detected maxima (circles) series of image pairs. The circles denoted the detected local maxima in the signal. To refine the estimate of the location of the maximum, a three-point peak fit is applied.

For some experiments, no clear result could be obtained using this method (due to either a lower image quality or very high spatial gradients). As an alternative approach, the height (rather than the location) of the displacement peak in the cross-correlation result can be used. For larger displacements, the correlation peak will be lower due to inplane loss of tracer pairs (Keane and Adrian 1992). Therefore, the reciprocal value of the correlation peak height can be used as alternative for the displacement.

\subsubsection{Image sorting}

Once the locations of the maxima have been determined, a value of the phase $\phi$ is assigned to each image pair. This phase corresponds to the location within the cardiac cycle, with $\phi=0$ corresponding to systole. For each image pair, the location of the nearest maxima are determined. Subsequently, the phase is determined by interpolation between $\phi=0$ and $\phi=1$ (which represent the positions of the two nearest maxima). This method compensates for small changes in the heart beat rate which would otherwise severely complicate the ensemble-averaging procedure. Once the phases have been assigned to the image pairs, they are grouped: all image pairs with a phase $\phi_{i}<\phi$ $<\phi_{i+1}$ are assigned to group $i$. The choice for the group spacing is a compromise between temporal resolution and the required number of images. From analyzing a large data of 5,000 images with varying group spacing, it was found that ten phase groups are sufficient to capture the characteristic cardiac cycle. For similar conditions, Vennemann et al. (2006a) found that 50 image pairs per group gave good results (while more image pairs hardly improved the results). This means that for each experiment, at least 500 image pairs are required.

The resulting phase assignments are also checked for any irregularities (either due to e.g. arrhythmia or resulting from a spurious phase estimation), by evaluating the variation of the distance between maxima. Data sets were only used if these irregularities were absent, so that quasi-periodicity could safely be assumed.

\subsubsection{Ensemble-averaging particle image velocimetry}

The velocity is calculated for each phase group using an inhouse PIV code. In this code, the cross-correlation data for each interrogation location are averaged (rather than the velocity fields) for all image pairs in each group; this results in a higher signal-to-noise ratio (Meinhart et al. 2000). The analysis is repeated in three iterations, each with a smaller interrogation area size (typically $64 \times 64$, 
$32 \times 32$ and $16 \times 16$ pixels, $50 \%$ overlap). The final interrogation area size-i.e. spatial resolution-depends on the data quality and total number of image pairs available. In between these steps, the data is validated and smoothed to reduce the influence of outliers during the iterations. The average of all images (also shown in the background of Fig. 8) is used to define a mask using a simple gray value threshold. This avoids spurious vectors outside of the vessels, but also shortens the calculation time significantly. Additionally, the mask can be used for visualization purposes.

Figure 6 shows the convergence of the ensemble averaging process for three different experiments. In this graph the differences between results obtained using $i$ and $i-1$ image pairs are shown. The average of the absolute deviation of all vectors in the measurement domain is calculated for each image pair that is added to the ensemble average. In the figure it can be seen that after 30-40 image pairs, adding extra measurement data to the ensemble average leads to changes in the displacement that are typically less than 0.05 pixel per vector. Note that typical displacements are of the order of 5-10 pixels, so that the variations are of the order of $1 \%$.

\subsubsection{Post-processing}

The velocity vector fields resulting from the ensemble-PIV procedure are validated using the universal outlier detection algorithm as described by Westerweel and Scarano (2005). Missing data, maximally a few percent of the total vector field in the masked area, is filled using bi-linear interpolation. The last part of the post-processing consists

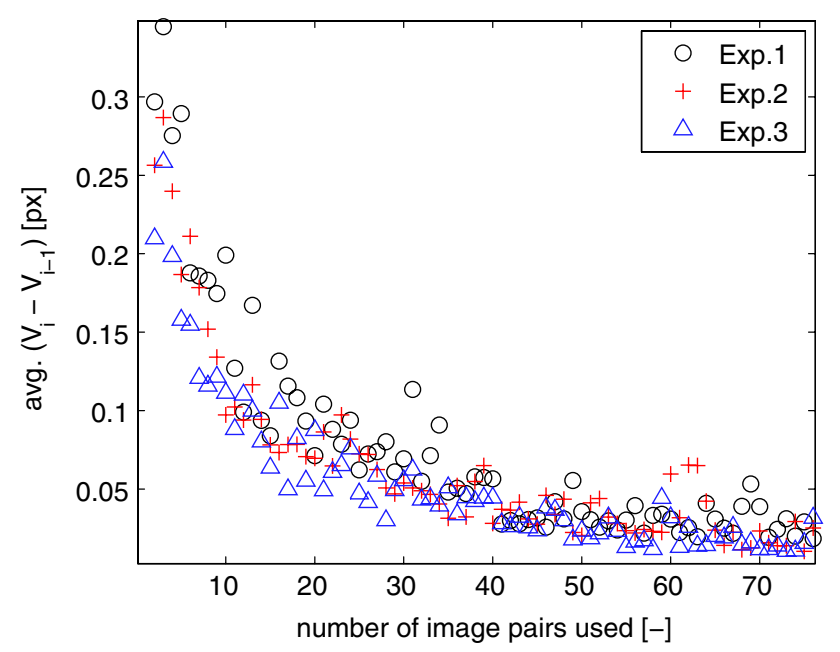

Fig. 6 Convergence of the data as a function of the number of image pairs for three different experiments; shown is the average difference in pixels between vector fields using $i$ and $i-1$ image pairs for the ensemble correlation of the calculation of the local wall shear stress, described in the following section.

\subsection{Calculation of the wall shear stress}

For the wall shear stress, the most conventional approach is to fit a flow profile to the data along a line perpendicular to the flow direction. Note that this reduces to Eq. 1 when a parabolic function is chosen for the fit. For the Reynolds and Womersley numbers of the flows under investigation, this choice can easily be defended $(R e<1$, Wo $\approx 0.2$, using $\mu=0.003 \mathrm{~Pa} \mathrm{~s}, d=100 \mu \mathrm{m}, f=2 \mathrm{~Hz}$ and $V=1 \mathrm{~mm} / \mathrm{s}$ as typical values for the largest vessels). The low Reynolds number will also lead to negligible short inlet lengths, as these scale as $L / D \approx 0.06 R e$ for laminar flows (p. 347 White 2007). Nevertheless, we encounter complications with this approach: to obtain values for the shear stress along a curved vessel, we need to first find the orientation of the vessel. This will then allow us to transform the data from their original measurement coordinate system (a cartesian coordinate system, expressed in PIV interrogation locations $x$ and $y$ ) to a coordinate system that is aligned with the vessel orientation (cylindrical coordinates, $r$ for radial position and $s$ for downstream distance). Figure 7 schematically shows these two coordinate systems. Finding the (local) coordinate transformation may seem trivial for a single, straight blood vessel-it reduces to a simple rotation. In practice, no standard automatic tools are available that can handle data of curved vessels with diameter changes and bifurcations (as seen in Figs. 8, 9). The only reliable method to achieve the wall-normal velocity profiles is to manually specify the orientation. Obviously, this

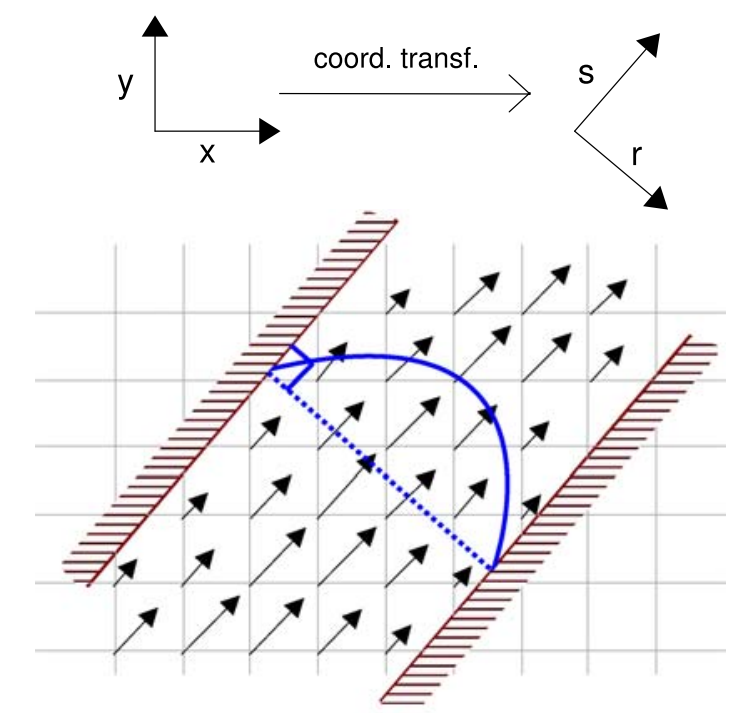

Fig. 7 The measurement coordinate system and the local vessel geometry coordinate system 


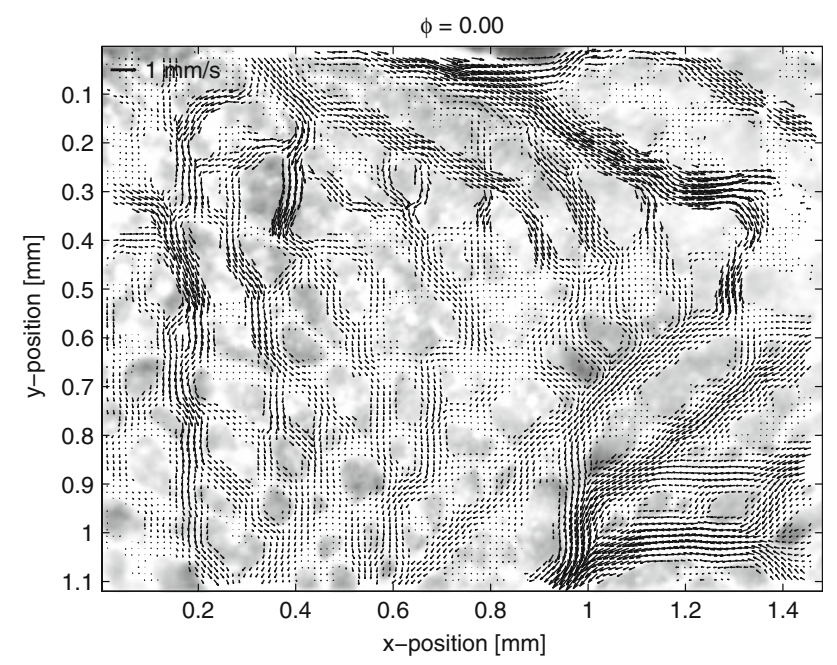

Fig. 8 Snapshot of a vitelline network at $6 \times$ magnification. The vectors represent the instantaneous blood flow velocity. The average image is shown in the background to indicate the complex geometry

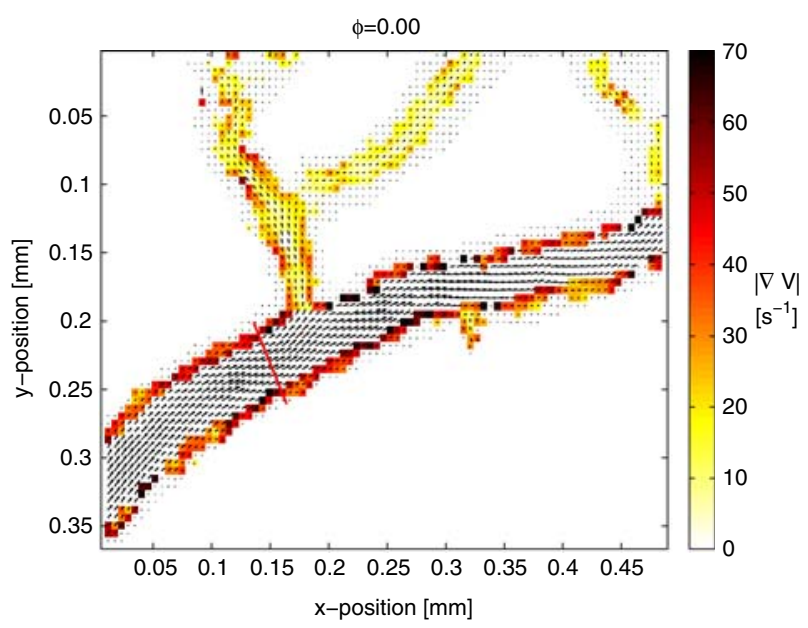

Fig. 9 Snapshot of a vitelline artery with bifurcations at $18 \times$ magnification. The false colors represent the total gradient of the velocity field (only the values at the wall are shown)

limits a systematic analysis of significant amount of data. A further complication results from the fact that the orientation in e.g. bifurcations cannot be defined easily.

As an alternative, a method is introduced to find the wall shear stress that only uses the data on the cartesian measurement coordinate system. In this method, the wall shear stress is derived directly from the measurement data using its definition, Eq. 2. On first sight, this would lead to the same problem as mentioned above: we still need the orientation of the wall-normal vector $n$, which is also required to find the streamwise component $u$ of the velocity vector. However, with two assumptions, this problem can be circumvented:
1. The flow close to the vessel wall can be assumed to be parallel to the wall. Therefore, the magnitude of the velocity vector will be equal to the streamwise component, i.e. $u \approx v_{\mathrm{abs}}=\left(v_{x}^{2}+v_{y}^{2}\right)^{1 / 2}$.

2. The flow can be assumed to be fully developed, as is evident from the negligible inlet lengths. This means that the derivatives in the wall-normal direction are significantly larger than the streamwise derivatives. In other words: the gradient vector will be dominated by the wall normal contribution.

As a consequence, the gradient required for the wall shear stress can be estimated from the magnitude of the spatial gradient:

$\frac{\partial u}{\partial n} \approx \sqrt{\left(\frac{\partial\left(v_{x}^{2}+v_{y}^{2}\right)^{1 / 2}}{\mathrm{~d} x}\right)^{2}+\left(\frac{\partial\left(v_{x}^{2}+v_{y}^{2}\right)^{1 / 2}}{\mathrm{~d} y}\right)^{2}}$

The advantage of Eq. 3 is that it uses the measurement coordinate system, rather than the significantly more complex system based on the local vessel geometry. In this study, Eq. 3 is evaluated using a central difference scheme. One drawback of this method is that the sign of the WSS is lost. The sign of the WSS can be relevant in the case of temporary flow reversal or backflow at the wall. In the current case, visual inspection of the velocity fields showed no such events. In later developmental stages, these may become relevant and sign information may be required. The proposed total gradient method can be extended to provide the sign information, for instance by specifying the orientation angle of each blood vessel segment. This way, the gradient can be calculated in the flow coordinate system, rather than the magnitude only, so that the sign is conserved. However, this will require either user input or some more sophisticated image processing to find the medial axis of each vessel segment.

\subsection{Extension to three-dimensions: scanning PIV}

While the vitelline network is mostly confined to a plane, there still may be three-dimensional effects (e.g. vessels that cross over each other). For a true quantitative study, e.g. to study perfusion, it may be necessary to document more than a single measurement plane. The methodology described above can easily be extended to a three-dimensional technique by repeating the steps at different $z$-locations. This is implemented using the computercontrolled translation stage of the microscope. The spacing between the $z$-locations $(\delta z=12 \mu \mathrm{m})$ is chosen to be comparable to the correlation depth of the measurement system (Olsen and Adrian 2000). This way, oversampling in the $z$ direction is avoided, as only particles within the correlation depth contribute to the correlation result. Note 
that the translation only takes place after the complete series of images has been recorded in a plane. The data is processed in an identical manner as the two-dimensional case. By visual inspection of the images, it is ensured that the embryo has not moved (this would result in a 'kink' in the reconstructed 3D data). Data sets with show any signs of movement are discarded.

The same reasoning as used for the derivation of Eq. 3 holds for the three-dimensional case. Extending the equation to incorporate the additional derivative (i.e. $\partial \cdots / \partial z$ ) is straightforward.

\section{Results and discussion}

\subsection{Single-plane measurements}

Two examples of snapshots (both at peak systole, $\phi=0$ ) are shown in Figs. 8 and 9. Figure 8 shows a distal region using $6 \times$ magnification. As background of the figure the average raw image is shown to visualize the tortuous network geometry. The data is masked using a threshold of the velocity/displacement in this case, ${ }^{3}$ rather than a mask based on the average raw image. The spatial resolution (i.e. vector spacing) of this measurement is approximately $13 \mu \mathrm{m}$, with a total field-of-view of $1.5 \times 1.1 \mathrm{~mm}^{2}$. These results were obtained with a final PIV iteration using 12 $\times 12$ pixel interrogation areas and $50 \%$ overlap. Figure 9 was obtained using $18 \times$ magnification and shows a larger vitelline artery with a number of bifurcations. In this figure, the total derivative-as obtained using Eq. 3-is shown as false colors at the location of the wall. The location of the wall is approximated by using a threshold of the velocity. The vector spacing is approximately $12 \mu \mathrm{m}$, with a $0.49 \times 0.37 \mathrm{~mm}^{2}$ field-of-view. Here a final PIV iteration with $32 \times 32$ pixel interrogation areas with $50 \%$ overlap was used. As can be seen, the threefold increase in magnification did not result in a similar increase in resolution. With a higher magnification, larger interrogation areas are needed to ensure a sufficient number of tracer particles in each area. A higher spatial resolution can be achieved by increasing either the number density of tracers particles or the total number of image pairs acquired.

To give an indication of the temporal and spatial resolution, Fig. 10 shows the evolution of the velocity throughout the cardiac cycle along a line as indicated in Fig. 9. To emphasize the periodic nature of this data, the cycle is repeated three times.

\footnotetext{
${ }^{3}$ By trial and error, we found that a tracer particle displacement of 0.2 pixel is a good threshold for most measurements; this corresponds to 0.03 and $0.02 \mathrm{~mm} / \mathrm{s}$ for Figs. 8 and 9 .
}

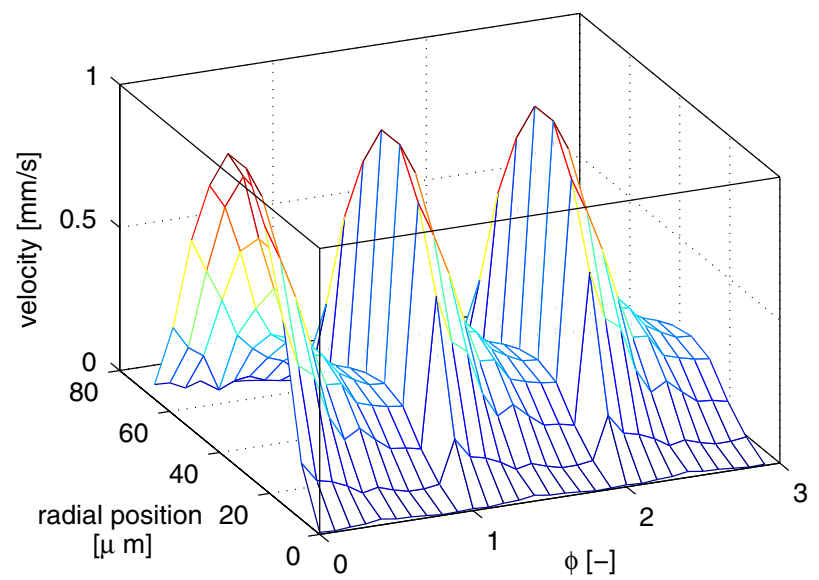

Fig. 10 Evolution of the velocity along the line indicated in Fig. 9. For clarity, the cycle is repeated three times

As mentioned in Sect. 2.3, the local wall shear stress can be determined from this data in two ways: by means of a fit to the velocity profile or directly from the total gradient at the wall. In the first method, a parabolic fit is used to determine both the location of the walls and the derivative at these points. The location of the wall-obtained from the zero-crossing of the fit-is more or less constant during the cycle, with a standard deviation of the order of $1 \mu \mathrm{m}$. The location of the wall can here be determined with greater accuracy than can be obtained by means of the thresholding method described earlier-the resolution of the latter is limited to the vector spacing $(12-13 \mu \mathrm{m}){ }^{4}$ The lack of variation in the location of the wall indicates that vessel compliance does not play a role in the blood vessels under investigation.

The results for the calculated gradients are shown in Fig. 11; again, the cycle is repeated three times to highlight the periodic character. Also shown in this figure is data obtained directly from the total gradients at the wall (cf. Fig. 9). The agreement between the two methods is good, except for the underestimation of the maximum by $15 \%$ (total gradient method compared to the profile method). Similar agreement was found for other profiles. Most likely, the underestimation is due to the fact that the spatial resolution is still insufficient for a more accurate determination of the gradients. The profile method uses more data points (typically 10-15, compared to the 3 for a central difference scheme), with higher signal-to-noise ratios due to the larger displacements away from the wall. Therefore, an overestimation of the profile method seems unlikely.

Despite the small underestimation, the total gradient method is significantly more efficient and easier to

\footnotetext{
${ }^{4}$ In theory, also the thresholding method could achieve a higher resolution for the wall location by interpolation, but measurement noise prevents this in practice.
} 


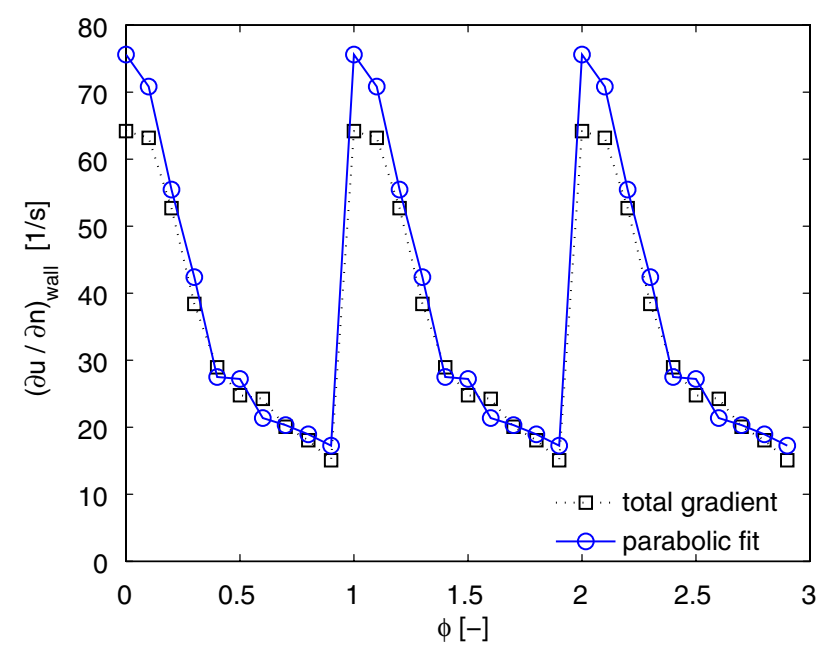

Fig. 11 The gradient at the wall as a function of the cardiac phase. Circles represent data obtained from a fit to the velocity profile (cf. Fig. 10.); squares represent data obtained directly from the total gradient

implement than the profile-fitting method. The latter process is rather laborious due to the complex geometry of the vitelline network. Note that Fig. 11 reports the shear rate, rather than the actual wall shear stress. To obtain the WSS, the data in the figure needs to be multiplied with the viscosity. For the viscosity of blood, usually a value of $3 \times$ $10^{-3} \mathrm{~Pa} \mathrm{~s}$ is used. The exact value depends on numerous parameters, including developmental stage and blood vessel diameter. Furthermore, the viscosity is dependent on the shear rate. With a known constitutive equation, incorporating the effects of shear thinning in the method based on Eq. 2 is straightforward. For the scope of this study, however, we assume that local differences in the WSS are dominated by changes in the gradients, rather than changes in the viscosity.

\subsection{Scanning measurements}

Figure 12 shows a visualization of the reconstructed threedimensional velocity field obtained in a scanning measurement. The same vessel network is used as shown in Fig. 9. Note that the orientation has been rotated by $90^{\circ}$ for visualization purposes. Sixteen planes are measured with a vertical spacing $\delta z=12 \mu \mathrm{m}$, comparable to the in-plane resolution. In the figure, an isosurface is shown of 0.3 pixels total displacement. For this visualization, the data has been smoothened twice by means of a $3 \times 3$ median filter to reduce the noise level. In theory, an isosurface of zero displacement would visualize the location of the vessel walls. However, the noise in the measurements close to the wall prevents this exact method. Note that the value of 0.3 is still small compared to the maximum values ( $8-10$ pixels at the centerline of the big vessels). Some streamlines have been calculated by integrating the (mean) velocity field to indicate the general flow pattern. The large middle vessel as seen in Fig. 12 extends beyond the measured $z$ range, hence the missing 'ceiling' of the vessel. The smaller, 'vertical' vessel on the left-hand-side is only present in four vertical planes. This highlights the need of such three-dimensional measurements if transport phenomena in these vessel networks are to be studied quantitatively. Even though parts of the vessels are missing in Fig. 12, estimating the flow, wall shear stress or other parameters can be done with more confidence than based on single plane measurements. For instance, a two-dimensional fit using a slice of a vessel perpendicular to the main flow direction (see e.g. Fig. 13) will give a more reliable estimate than one based on a onedimensional profile. For the latter, it is usually implicitly assumed that it is taken in the midplane of the vessel (thus capturing the maximum of the flow). With the scanning measurement results, this uncertainty is obviously removed: the data shown in Fig. 9, for instance, has been selected from the total set of measurements and was found to be the center plane of the large vessel.

The vitelline arteries and veins are more or less aligned with the measurement plane, so most of the displacement is expected to be in-plane. Ideally, all three velocity

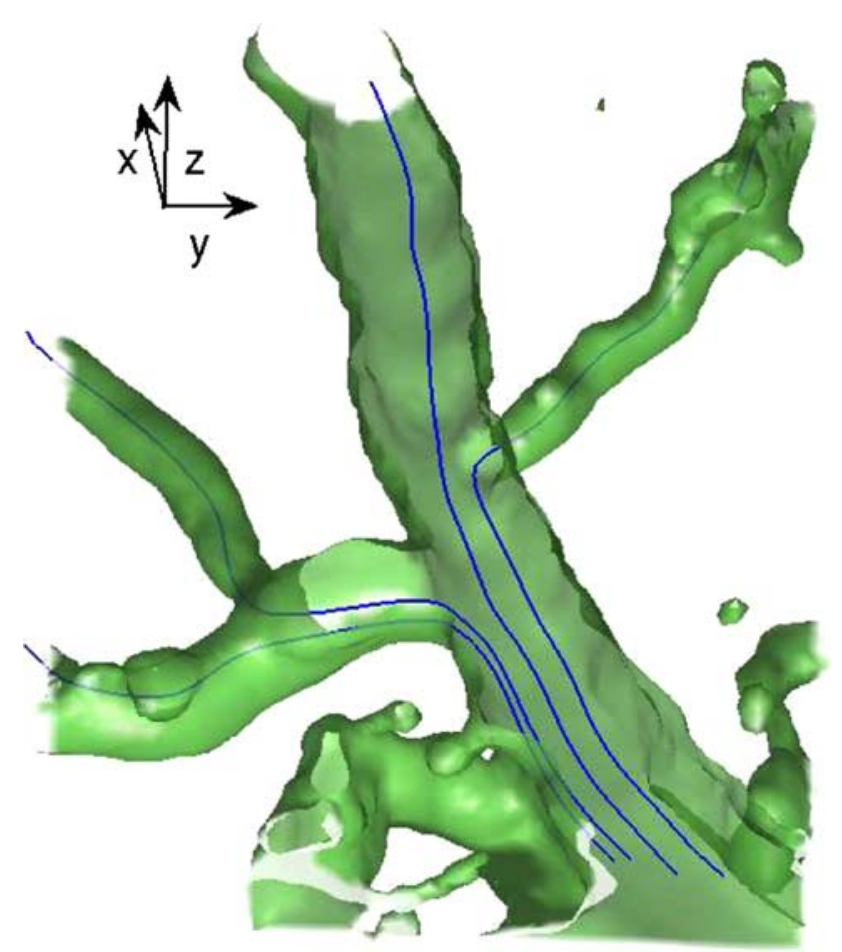

Fig. 12 Three-dimensional visualization of the data obtained from a scanning PIV measurement. Shown is an isosurface of 0.3 pixel displacement at $\phi=0.1$. Some streamlines have been calculated to indicate the general flow pattern. Note that the orientation has been rotated by $90^{\circ}$ with respect to Fig. 9 


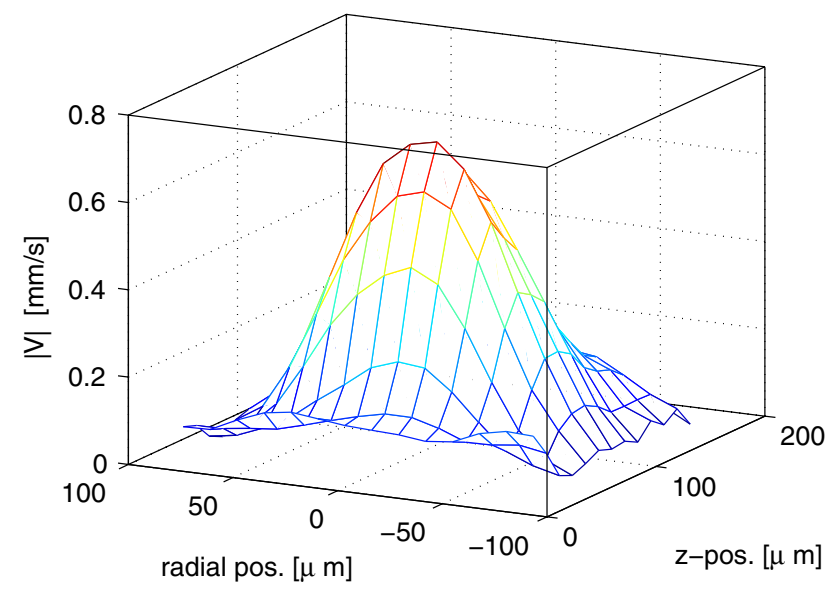

Fig. 13 Two-dimensional flow velocity profile in a slice perpendicular to the flow direction in a vitelline artery at systole $(\phi=0)$. The slice is taken from the same data set as shown in Fig. 12, along the red line in Fig. 9. The magnitude of the local velocity is shown, the radial position refers to the location along the red line of Fig. 9

components would be available. This can be achieved by e.g. stereoscopic micro-PIV (Lindken et al. 2006). However, in the current experiment an accurate calibration as required by this technique was difficult to achieve with the time-sensitive measurements. Alternatively, one could estimate all three velocity components from the current $3 \mathrm{D}-2 \mathrm{C}$ data using the continuity equation, as demonstrated by e.g. Bown et al. (2007). We are intending to use this approach in future studies.

\subsection{Repeatability}

For physiological studies, it is important that the experiment can be repeated after a certain amount of time. During and in between measurements, the embryo needs to be kept at a constant temperature by (partially) immersing them in a constant temperature water bath. Furthermore, they should be properly sealed to avoid excessive evaporation of fluids. By doing so, we managed to repeat the experiments as shown in Fig. 8 (with the same embryo and field-of-view) after intervals of 60 min up to $7 \mathrm{~h}$. A small portion of tracer material is lost due to adhesion to the endothelium, but extra tracer particles can easily be injected if needed. Therefore, the methodology described in this manuscript is suitable for the study of physiological changes - either due to development or due to mechanical or chemical intervention.

\section{Conclusions and outlook}

Using microscopic particle image velocimetry, it is possible to determine the local wall shear stress in vivo in a repeatable manner. This is done by first reconstructing instantaneous blood flow velocity fields using correlation ensemble averaging PIV in combination with a phasesorting algorithm. From these velocity fields, the shear stress can be derived in two methods: either from a fit to the velocity profile along the vessel diameter or from the total gradient. The former is difficult to automate due to the complex geometry of the vascular network. The latter is computationally much more efficient and easy to implement. Both methods give comparable results, except for an underestimation by $15 \%$ at peak systole for the total gradient method. Most likely, this is caused by an insufficient spatial resolution of the velocity field. Despite the underestimation, the total gradient technique will be useful in the study of the interaction between hemodynamics and the surrounding tissue. Especially in complex segments (e.g. bifurcations), fitting a velocity profile is not practical, while the total gradient method provides detailed, local information with a typical resolution of $12 \times 12 \mu \mathrm{m}^{2}$.

We intend to use the technique described in this paper to perform quantitative studies of the effect of mechanical and/or biochemical interventions in the vitelline network. For such a quantitative analysis, we need the threedimensional velocity field, rather than a single measurement plane. As shown in this manuscript, a scanning micro PIV measurement can provide this information.

While we have here applied the technique to the vitelline network, many other applications are possible. Any flow with optical access can in principle be measured, as long as tracer particles can be introduced. Within the chick embryo, the chorioallantoic membrane is another commonly-used model system for the study of e.g. angiogenetic processes (Borges et al. 2003). Other applications include perfusion and vascularization studies in tumors using skinfolds in mice (Lehr et al. 1993). A final example of a challenging application is the study of retinal angiogenesis and hemodynamics (Gariano and Gardner 2005).

Acknowledgements $P$. Vennemann received support through the Dutch Technology Foundation (STW). The authors would like to thank E. Bon (Erasmus MC, Rotterdam) for her assistance during the measurements.

Open Access This article is distributed under the terms of the Creative Commons Attribution Noncommercial License which permits any noncommercial use, distribution, and reproduction in any medium, provided the original author(s) and source are credited.

\section{References}

Bellairs R, Osmond M (2005) The atlas of chick development. Elsevier Academic Press, Amsterdam

Borges J, Tegtmeier FT, Padron NT, Mueller MC, Lang EM, Stark GB (2003) Chorioallantoic membrane angiogenesis model for tissue engineering: a new twist on a classic model. Tissue Eng 9(3):441-450 
Bown MR, MacInnes JM, Allen RWK (2007) Three-component micro-PIV using the continuity equation and a comparison of the performance with that of stereoscopic measurements. Exp Fluids 42(2): 197-205

Chien S (1970) Shear dependence of effective cell volume as a determinant of blood viscosity. Science 168:977-979

Gariano RF, Gardner TW (2005) Retinal angiogenesis in development and disease. Nature 438(7070):960-966

Hamburger V, Hamilton HL (1951) A series of normal stages in the development of the chick embryo. J Morphol 88(1):49-92

Hierck BP, van der Heiden K, Poelma C, Westerweel J, Poelmann RE (2008) Fluid shear stress and inner curve remodeling of the embryonic heart. choosing the right lane! Sci World J (revision submitted)

Hogers B, De Ruiter MC, Grittenberger-de Groot AC, Poelmann RE (1997) Unilateral vitelline vein ligation alters intracardiac blood flow patterns and morphogenesis in the chick embryo. Circ Res 80:473-481

Janmey PA, McCulloch CA (2007) Cell mechanics: integrating cell responses to mechanical stimuli. Annu Rev Biomed Eng 9:1-34

Keane RD, Adrian RJ (1992) Theory of cross-correlation analysis of PIV analysis. Appl Sci Res 49:191-215

Kind C (1975) The development of the circulating blood volume of the chick embryo. Anat Embryol 147(2):127-132

Ku DN, Giddens DP, Zarins CK, Glagov S (1985) Pulsatile flow and atherosclerosis in the human carotid bifurcation. positive correlation between plaque location and low oscillating shear stress. Arterioscler Thromb Vasc Biol 5(3):293-302

Landau LD, Lifshitz EM (1987) Fluid mechanics. Pergamon Press, New York

Le Noble F, Moyo D, Pardanaud L, Yuan L, Djonov V, Mathijssen R, Brant C, Fleury V, Eichmann A (2004) Flow regulates arterialvenous differentiation in the chick embryo yolk sac. Development 132(2):361-375

Lee JY, Ji HS, Lee SJ (2007) Micro-PIV measurements of blood flow in extraembryonic blood vessels of chicken embryos. Physiol Meas 28(10):1149-1162

Lehr HA, Leunig M, Menger MD, Nolte D, Messmer K (1993) Dorsal skinfold chamber technique for intravital microscopy in nude mice. Am J Pathol 143(4):1055-1062

Lindken R, Westerweel J, Wieneke B (2006) Stereoscopic micro particle image velocimetry. Exp Fluids 41(2):161-171

McCormick SM, Eskin SG, McIntire LV, Teng CL, Lu CM, Russell CG, Chittur KK (2001) DNA microarray reveals changes in gene expression of shear stressed human umbilical vein endothelial cells. Proc Natl Acad Sci 98(16):8955

McDonald DA (1974) Blood flow in arteries. Edward Arnold, London

Meinhart CD, Wereley ST, Santiago JG (2000) A PIV algorithm for estimating time-averaged velocity fields. J Fluids Eng 122(2):285-289
Olsen MG, Adrian RJ (2000) Out-of-focus effects on particle image visibility and correlation in microscopic particle image velocimetry. Exp Fluids 29:166-174

Popel AS, Johnson PC (2005) Microcirculation and hemorheology. Annu Rev Fluid Mech 37(1):43-69

Reneman RS, Arts T, Hoeks APG (2006) Wall shear stress-an important determinant of endothelial cell function and structurein the arterial system in vivo; discrepancies with theory. J Vasc Res 43(3):251-269

Resnick N, Yahav H, Shay-Salit A, Shushy M, Schubert S, Zilberman LC, Wofovitz E (2003) Fluid shear stress and the vascular endothelium: for better and for worse. Progr Biophys Mol Biol 81:177-199

Sugii Y, Okuda R, Okamoto K, Madarame H (2005) Velocity measurement of both red blood cells and plasma of in vitro blood flow using high-speed micro PIV technique. Meas Sci Technol 16(5):1126-1130

Topper JN, Gimbrone MA Jr. (1999) Blood flow and vascular gene expression: fluid shear stress as a modulator of endothelial phenotype. Mol Med Today 5(1):40-46

Van der Heiden K, Groenendijk BC, Hierck BP, Hogers B, Koerten HK, Mommaas AM, Gittenberger-de Groot AC, Poelmann RE (2006) Monocilia on chicken embryonic endocardium in low shear stress areas. Dev Dynam 235(1):19

Vennemann P, Kiger KT, Lindken R, Groenendijk BCW, Stekelenburg-de Vos S, Ten Hagen TLM, Ursem NTC, Poelmann RE, Westerweel J, Hierck BP (2006a) In vivo micro particle image velocimetry measurements of blood-plasma in the embryonic avian heart. J Biomech 39:1191-1200

Vennemann P, Lindken R, Hierck BP, Westerweel J (2006b) Volumetric particle image velocimetry in the developing chicken heart. J Biomech 39:S616

Vennemann P, Lindken R, Westerweel J (2007) In vivo whole-field blood velocity measurement techniques. Exp Fluids 42:495-511

Wagman AJ, Hu N, Clark EB (1990) Effect of changes in circulating blood volume on cardiac output and arterial and ventricular blood pressure in the stage 18, 24 and 29 chick embryo. Circ Res 67:187-192

Weinbaum S, Zhang X, Han Y, Vink H, Cowin SC (2003) Mechanotransduction and flow across the endothelial glycocalyx. Proc Natl Acad Sci 100(13):7988-7995

Westerweel J (2008) On velocity gradients in PIV interrogation. Exp Fluids. doi:10.1007/s00348-007-0439-3

Westerweel J, Scarano F (2005) Universal outlier detection for piv data. Exp Fluids 39(6): 1096-1100

White FM (2007) Fluid mechanics, 6th edn. Elsevier Academic Press, Amsterdam

Willert CE (1997) Stereoscopic digital particle image velocimetry for application in wind tunnel flows. Meas Sci Technol 8:1465-1479 